\title{
A Comparison of Rectal Suction and Full Wall Biopsy in Hirschsprung's Disease
}

\author{
Sofie Örnö Ax, E. Arnbjörnsson, D. Gisselsson-Nord \\ Lund University Hospital, Department of Paediatric Surgery, Lund, Sweden \\ Email: sofie.orno.ax@gmail.com
}

Received November 9, 2013; revised December 8, 2013; accepted December 16, 2013

Copyright (C 2014 Sofie Örnö Ax et al. This is an open access article distributed under the Creative Commons Attribution License, which permits unrestricted use, distribution, and reproduction in any medium, provided the original work is properly cited. In accordance of the Creative Commons Attribution License all Copyrights (C) 2014 are reserved for SCIRP and the owner of the intellectual property Sofie Örnö Ax et al. All Copyright (C) 2014 are guarded by law and by SCIRP as a guardian.

\begin{abstract}
Introduction: Hirschsprung's disease (HD) is defined as congenital agangliosis of the colon wall from the rectum extending cranially. There are several radiologic methods for screening for HD but it is a biopsy of the colon wall that confirms the diagnosis. Initially the full wall (FW) biopsy was the method of choice. FW biopsy requires general anaesthesia and creates a full wall laceration of the colon wall. The newer method called rectal suction (RS) biopsy is carried out by means of a catheter inserted into the rectum that blindly cuts a biopsy including mucosa and submucosa. It can be performed bedside. If ganglion cells are seen in the biopsy specimen, HD can be excluded. The question that arises is: Can we safely move on from FW biopsy to RS biopsy without compromising diagnostic accuracy? Method and Material: Our study is a retrospective revision of 20 FW biopsies that were supplemented with RS biopsies. RS biopsies with insufficient submucosa were excluded. The RS biopsies were assessed by a senior pathologist in a double blind fashion and the result was compared with FW biopsies. FW biopsy was considered gold standard. Results: The exact binominal test for the non-inferiority test of congruence of RS biopsy with FW biopsy as gold standard was not statistically significant using an inferiority margin of $80 \%(p=0.06918)$ [H0: p_RS $<0.8$, HA: $p \_R S \geq 0.8$ ]. The estimated probability for a successful RS biopsy was $95 \%$ and the one-sided $95 \%$-confidence interval associated with the test was 0.784 to 1.00. Conclusion: Our results indicate that we can move on from FW biopsy to RS biopsy without compromising the diagnostic value of the rectal biopsy in a clinically significant way. A prospective study to confirm our results would be of great value.
\end{abstract}

\section{KEYWORDS}

Hirschsprung; Biopsy; Rectal; Techniques

\section{Background \& Introduction}

Hirschsprung's disease (HD) is a congenital intestinal motility disorder characterized by absent gastrointestinal ganglia cells and neural cell hyperplacia affecting distal parts of the intestine. The absence of the neural controlling ganglia cells results in affected parts of the bowel being unable to support peristalsis, absent recto-anal inhibitory reflex (RAIR) and marked dilatation of the ganglionic segment proximal to the affected bowel [1]. HD has an incidence of 1 of 5000 live births; $80 \%$ of those affected are male. Associated congenital anomalies and linked diseases such as Down syndrome, colonic and ileal atresia, congenital heart disease and a variety of syndromes characterized by abnormal development of ganglion precursor cells are seen in 10\% - 15\% of HD patients [2].

In the common HD case the agangliotic segment starts adjunct to the linea dentate, and is limited to the rectum or sigmoid colon. Only $20 \%$ of affected children are not discovered during their first year of life. If constipation occurs later than 4 weeks after birth and the patient is previously healthy it is suggested that HD is so unlikely that investigating with a rectal biopsy is unnecessary [3]. Delayed diagnosis, even as short as one week, significantly increases the risk of serious complications in neonatal patients [4]. If HD is treated according to current 
protocols the outcome for the patients is generally good.

The classic microscopic features of HD are absence of ganglion cells and abundant hypertrophic cholinergic nerves of the extrinsic parasympathetic nervous system. Both the myenteric and submucous plexuses are agangliotic. At any given level, agangliosis of both plexuses is synchronous. A biopsy specimen must be taken well over the dentate line to make sure the material represents colon and not the anal channel, which is innately agangliotic.

The rectal full wall biopsy (FW biopsy) was the only option for a histological diagnosis until the mid-1960s. FW biopsy provides a specimen where both the muscularis mucosae and muscularis propria are visible. The amount of material from a full wall biopsy is abundant for slicing and staining for histological diagnosis. The drawbacks are that the procedure is invasive and the patient must undergo general anaesthesia. It makes a full thickness laceration of the colon wall, requires stitching and often some minor bleeding is involved. It can give rise to infection, re-perforation and other complications associated with surgical manoeuvres in the intestine [5].

In 1965 Dobbins and Bill demonstrated that ganglion cells could be reliably and consistently seen on rectal suction biopsies (RS biopsies) merely containing the same depth of submucosa as the depth of mucosa above it. This became clinically useful when it was proven that the presence of ganglion cells in the submucous plexus rules out aganglionosis; thus biopsies containing an adequate amount of agangliotic submucosa can confirm HD $[6,7]$.

RS biopsy is a bedside procedure that requires no general anaesthesia or suturing. It is performed via a catheter inserted into the rectum that blindly clutches and cuts a small piece of the colon wall via a small vacuum created at its tip.

There are many RS biopsy devices available on the market and in many centres the RS biopsy has completely replaced the FW biopsy as a method of choice; it is a gentle and quick procedure that can be repeated if the material is insufficient.

Adjustments such as specifically designed instead of multipurpose instruments, constant (machine created) suction and exchangeable blades have lowered the ratio of insufficient tissue from $25 \%$ to $7 \%$ [8-10]. Confirmation of the diagnosis HD with RS biopsy has an evidence rating with a strength of $\mathrm{C}$ in the Strength of Recommendation Taxonomy (SORT) [11]. Bowel perforation and rectal haemorrhage are the two most serious complications of RS biopsy; fortunately they are extremely rare. Rees et al. studied a series of 1340 RS biopsies; there were 6 complications of which 3 were bowel perforations $[12,13]$.

The diagnosis of agangliosis is a negative finding.
With better and faster treatment a rapid, reproducible and accurate biopsy method for histological diagnosis is of the uttermost importance. Can we move on from FW biopsy to RS biopsy without increasing the risk of false positive HD outcomes?

\section{Method \& Materials}

Our study is a retrospective survey of rectal suction biopsy specimens retrieved from paediatric patients with suspected HD at Skåne University Hospital, Lund, Sweden. All cases included were performed during general anaesthesia and the procedures were carried out in theatre. A consultant surgeon performed the RS biopsies with a C-2rbi ${ }^{\circledR}$ device. Two or three RS biopsy samples were collected during each session. The RS biopsy session was instantly followed by a FW biopsy.

The biopsy specimens were then embedded, stained according to current staining protocols at the time and mounted on separate slides. The immunostains Calretinin, S100, Neuron Specific Enolase (NSE) and Acetylcholine Histochemistry (AChT) were used in addition to the traditional hematoxylin-eosin (H\&E) stain. A pathologist assessed the biopsies and a note was made to describe the findings and confirm or discard HD. In our study, this evaluation of the FW biopsy and subsequent operations were considered gold standard in terms of agangliosis or agangliosis of the specimen. In the 4 agangliotic FW biopsy specimens the agangliosis was confirmed by specimens taken intraoperatively when the patients were being surgically treated for HD.

The included cases underwent a brief microscopical preview examination to confirm that the material was the RS biopsy specimen and that submucosa was visible. The slides containing RS biopsy specimens were specified on the referral note. When the RS biopsy specimens were unspecified, the RS biopsy material was selected during the preview session. Criteria for an accepted RS biopsy specimen were that it did not contain any tissue deeper than the submucosa, it was unmistakably smaller than the FW biopsy and that it was mentioned in the responding note that the RS biopsy material was embedded and reviewed.

After the preview session, the included cases were examined by Senior Consultant Pathologist Anna Måsbäck. The process was double-blind with anonymised RS biopsy slides. The examination was in two parts so that $\mathrm{H}$ \& E stained slides were reviewed first, after this $\mathrm{H}$ \& $\mathrm{E}$ and S100, NSE and/or AChT were evaluated together. If the specimen was stained with Calretin in this was viewed as an addition to the other stains as the final step of the evaluation.

The congruence of RS biopsy was compared with gold standard (FW biopsy). The congruence is defined as proportion of correctly classified cases. The exact binomial 
test was used to test the non-inferiority of the congruence of the RS biopsy-method in relation to the FW biopsymethod. The non-inferiority margin was set to 0.8 , i.e. the null hypothesis is that the accuracy of the RS biopsymethod is less than 0.8 , and if this null is rejected, we are willing to accept that the accuracy of the RS biopsymethod is non-inferior to the gold standard method. The probability of rejecting the null, when in fact it is true, is the $p$-value associated with the test; $p$-values $0.1>p>$ 0.01 are considered weak evidence, $0.01>p>0.001$ as moderately strong evidence, $p<0.001$ as strong evidence against the null [14]. The confidence intervals were calculated using the Clopper-Pearson method which is known to produce conservative confidence-intervals, i.e. the probability is at least $95 \%$. This method is called the exact confidence intervals, since it uses the binomial distribution and is based on the inversion of the test. This means that the lower CI reported here will be the highest probability that does not cover the observed value with the stated coverage probability. All analyses were performed using the free software R, ver. 3.0.0 (R Core Team, 2013) [15].

The sensitivity of the FW biopsy was 94\% and its specificity was $100 \%$ in diagnosing HD. Data regarding staining techniques and embedding was collected as an additional description of our method to empower its reproducibility and give increased insight in our retrospective study design. The descriptive data is displayed in Tables 1 and 2.

On 25 February 2010 permission was granted by the Regional Ethics Committee for our research group to carry out the trial. Our project title is "Surgical treatment and observing of children with congenital malformations or chronic diseases” and its ethics trial number 2010/49. This study is included in the project and it does not concern the Swedish law of "Research that concerns patient safety” (2003:460). All patient data was coded and patient integrity was respected throughout the entire process.

\section{Results}

From January 2007 to August 201265 patients from the paediatric population underwent rectal biopsy. Of these, 30 were patients with suspected HD and according to referral notes 23 patients in this group were investigated with both RS biopsy and FW biopsy on the same occasion. In 3 of the included cases the RS biopsy material was not embedded, for unknown reasons. The ages of our 20 included patients ranged from 8 days to 14 years (mean age 9 months, median age 7 months), 11 were male and 9 were female.

Of the 20 biopsies included, 4 (20\%) were agangliotic and 16 (80\%) presented with ganglion cells when the FW biopsy material was reviewed. One (5\%) of the RS biop-
Table 1. Distribution of included cases and IHC staining during the report period.

\begin{tabular}{ccccc}
\hline year & No. of biopsies & S100 + NSE & Calretinin & AChE \\
\hline $\mathbf{2 0 0 7}$ & 2 & 2 & 0 & 0 \\
$\mathbf{2 0 0 8}$ & 2 & 0 & 0 & 2 \\
$\mathbf{2 0 0 9}$ & 5 & 5 & 1 & 0 \\
$\mathbf{2 0 1 0}$ & 9 & 9 & 8 & 0 \\
$\mathbf{2 0 1 1}$ & 2 & 2 & 2 & 0 \\
total: & 20 & 18 & 11 & 2 \\
\hline
\end{tabular}

Table 2. Identification of ganglion cells with H\&E (haematoxylin-eosin) stain and IHC stains. All cases were examined in three sets: first $\mathrm{H} \& E$, then $H \& E$, NSE, S100 and ACtH, then H\&E, NSE, S100, ACtH and calretinin. When ganglion cells were noted this was recoded as if control stain for IHC had been performed.

\begin{tabular}{|c|c|c|c|c|c|}
\hline $\begin{array}{l}\text { Hirschsprungs } \\
\text { disease }\end{array}$ & $\begin{array}{c}\text { ganglion } \\
\text { cells: } \\
\text { FW } \\
\text { biospy }\end{array}$ & $\begin{array}{c}\text { ganglion } \\
\text { cells } \\
\text { H\&E: RS } \\
\text { biopsy }\end{array}$ & $\begin{array}{c}\text { ganglion cells } \\
\text { NSE, S100, } \\
\text { AChE: RS } \\
\text { biopsy }\end{array}$ & $\begin{array}{l}\text { ganglion } \\
\text { cells } \\
\text { calretinin: } \\
\text { RS biopsy }\end{array}$ & $\begin{array}{c}\text { control } \\
\text { stain, } \\
\text { IHC }\end{array}$ \\
\hline no & yes & yes & yes & yes & no \\
\hline no & yes & yes & yes & yes & no \\
\hline no & yes & yes & yes & yes & yes \\
\hline no & yes & yes & yes & yes & yes \\
\hline no & yes & yes & yes & yes & yes \\
\hline no & yes & yes & yes & yes & yes \\
\hline no & yes & yes & yes & yes & yes \\
\hline no & yes & yes & yes & not stained & no \\
\hline no & yes & yes & yes & not stained & no \\
\hline no & yes & yes & yes & not stained & no \\
\hline no & yes & yes & yes & not stained & yes \\
\hline no & yes & yes & no & yes & no \\
\hline no & yes & yes & no & not stained & yes \\
\hline no & yes & no & no & yes & yes \\
\hline no & yes & no & no & yes & yes \\
\hline yes & no & no & no & not stained & no \\
\hline yes & no & no & no & not stained & no \\
\hline yes & no & no & no & not stained & yes \\
\hline no & yes & no & no & no & no \\
\hline yes & no & no & no & no & yes \\
\hline
\end{tabular}

sies presented with no ganglion cells, but in its corresponding FW biopsy, ganglion cells were present (see Table 3). 
Table 3. Distribution of identified ganglion cells in FW and RS biopsies.

\begin{tabular}{cccc}
\hline Kolumn1 & FW biopsy (+) & FW biopsy (-) & Total: \\
\hline RS biopsy (+) & 15 & 0 & 15 \\
RS biopsy $(-$ & 1 & 4 & 5 \\
Total: & 16 & 4 & 20 \\
\hline
\end{tabular}

The exact binominal test for the non-inferiority test of congruence of RS biopsy with FW biopsy as gold standard was not statistically significant using an inferiority margin of $80 \%$ ( $p=0.06918)$ [H0: $\mathrm{p} \_\mathrm{RS}<0.8$, HA: P_RS $\geq 0.8]$. The estimated probability for a successful RS biopsy was 95\% and the one-sided 95\%-confidence interval associated with the test was 0.784 to 1.00 .

The descriptive data of the success rate for staining techniques and the use of staining protocols over time are displayed in Tables 1 and 2.

\section{Discussion}

RS biopsy can be performed without general anaesthesia; it is a fast and relatively comfortable procedure with a very limited record of complications. If the retrieved tissue does not contain a sufficient portion of submucosa a re-biopsy is taken. A re-biopsy has consequences such as diagnostic delay, longer hospital stay, a relatively higher cost of treatment, patient and parental anxiety and unnecessary work for the pathologist.

Our study investigates if the RS biopsy that has sufficient tissue to allow meaningful interpretation is comparable with the FW biopsy regarding strength of the histologic diagnosis. FW biopsy is golden standard, but that is not to say it has $100 \%$ sensitivity. The congruence of 0.8 was chosen as a lower limit based on how many rebiopsies it was felt could be accepted with a procedure that was still more beneficial than the FW biopsy. If a false positive result is suspected, common practice is to perform a re-biopsy. In a worst case scenario, where the significant accuracy is as low as 0.8 , the significant accuracy of a re-biopsy would be 0.95 which is acceptable for all practical purposes. Since RS biopsy has the advantage of being a safe and fast procedure it was agreed that this risk of a re-biopsy could be accepted and the RS biopsy would still be considered non-inferior to the golden standard test. To estimate if the true congruence was likely to be 0.8 or higher the non-inferiority test was applied.

The non-inferiority test gave a p-value of 0.06918 and the estimated probability for a successful RS biopsy was predicted to $95 \%$. From a clinical point of view the classification of our results as "significant" with a $p$-value of 0.05 is somewhat random [14]. Seen in the context that Hirschsprung's disease is by definition a histologic diagnosis and that regardless of whether the pathologist finds agangliosis it is still the severity of symptoms that deter- mine if surgery is required, we are pleased that our results show such promise. Our results indicate that a significant $(p<0.05)$ minimum $80 \%$ congruence of the RS biopsy with the FW biopsy can be expected in a slightly bigger study, considering that the estimated one-sided 95\%-confidence interval for a successful biopsy was 0.784 to 1.00 . This is very close to the interval 0.8 to 1.0 .

Our report has the obvious disadvantage of being a retrospective survey of a small cohort. In $40 \%$ of included cases the RS biopsy specimen slides were not specified and had to be chosen according to size and content. It was often not noted which RS biopsy that corresponded to the slide, just that it corresponded to one of the two or three RS biopsies from one particular case. In three cases, RS biopsy was clearly notified in the referral note but the material was not embedded. This was surprising because our custom is to embed and stain all biopsy material provided.

Even though our included RS biopsy cases had sufficient portions of submucosa and each case included specimens from two or three RS biopsies there is still a risk of missing ganglion cells. There is always a risk of a false positive diagnosis with both methods. We have chosen FW biopsy as gold standard in this study but the original diagnosis was reached based on the FW and the RS biopsies together. There is a possibility that the diagnosis was established predominantly from the RS biopsy material but in reality this is not likely to happen.

With an RS biopsy specimen the classic H\&E stain is, even for an experienced pathologist, difficult to interpret. The different IHC stains in our study proved quite troublesome because of out-dated methods and the frozen sections do not age well. In our material, when evaluating the $\mathrm{H} \& \mathrm{E}$ stained specimens, 3 out of 17 (18\%) gangliotic specimens were deemed agangliotic. With auxiliary stains this number decreased to 1 out of 17 (6\%). Due to the small size of our study population and the 4 different combinations of stains, we refrain from commenting on which of the auxiliary stains has been of the greatest help.

We would welcome a large prospective study of the RS biopsy with FW controls with open arms, but is this beneficial for the patients? The RS biopsy is a welldocumented and widespread method that shortens hospital stay and reduces patient suffering. It would perhaps not be ethically justifiable to continue with the FW biopsies to confirm that RS biopsies are safe. A prospective report on the diagnostic accuracy of RS biopsies, which includes sufficient tissue to allow meaningful interpretation and stained with current IHC methods, would be highly appreciated and a better choice moral-wise.

\section{Conclusion}

Our conclusion is that we can move on from FW biopsy 
to RS biopsy without compromising the diagnostic accuracy of the rectal biopsy in a clinically significant way. A prospective study of the RS biopsy with up-to-date ICH methods to confirm our results would be of great value.

\section{Acknowledgements}

Anna Måsbäck, Senior Staff Pathologist, Dept of Pathology, University and Regional Laboratories, Region Skåne, for guidance and incredible teaching skills.

Gillian Sjödahl, Lexis English for Writers, Lund Sweden, for linguistic revision of the manuscript.

Fredrik Nilsson, Biostatistician at the Competence Centre for Clinical Research, Skåne University Hospital, LUND, Sweden, for statistical advice and endless patience.

Lena Tran, Biomedical Scientist at the Department of Clinical Pathology, University Hospital of Skane, for introducing the wonders of immunohistochemistry.

\section{REFERENCES}

[1] K. Vult von Steyern, P. Wingren, M. Wiklund, P. Stenström and E. Arnbjörnsson, "Visualisation of the Rectoanal Inhibitory Reflex with a Modified Contrast Enema in Children with Susected Hirschsprung Disease,” Pediatric Radiology, Vol. 43, No. 8, 2013, pp. 950-957. http://dx.doi.org/10.1007/s00247-013-2622-4

[2] D. Spouge and P. A. Baird, "Hirschsprung Disease in a Large Birth Cohort,” Teratology, Vol. 32, No. 2, 1985, pp. 171-177. http://dx.doi.org/10.1002/tera.1420320204

[3] A. Ghosh and D. M. Griffiths, "Rectal Biopsy in the Investigation of Constipation," Archives of Disease in Childhood, Vol. 79, No. 3, 1998, pp. 266-268. http://dx.doi.org/10.1136/adc.79.3.266

[4] C. C. Lee, R. Lien, M. C. Chiang, P. H. Yang, S. M. Chu, J. H. Fu and J. Y. Lai, "Clinical Impacts of Delayed Diagnosis of Hirschsprung's Disease in Newborn Infants," Pediatrics \& Neonatology, Vol. 53, No. 2, 2012, pp. 133137. http://dx.doi.org/10.1016/j.pedneo.2012.01.011

[5] J. C. Langer, "50 Years Ago in the Journal of Pediatrics: Rectal Biopsy as an Aid in the Diagnosis of Diseases of Infants and Children,” Journal of Pediatrics, Vol. 162, No. 2, 2013, p. 301. http://dx.doi.org/10.1016/j.jpeds.2012.09.020

[6] E. Gilbert-Barness, R. P. Kapur, L. L. Oligny and J. R. Siebert, "Potter's Pathology of the Fetus, Infant and Child," 2nd Edition, Mosby Elesevier, Philadelphia, 2007, p. 1176.

[7] W. O. Dobbins and A. H. Bill, "Diagnosis of Hirschsprung's Disease Excluded by Rectal Suction Biopsy," The New England Journal of Medicine, Vol. 272, No. 19, 1965, pp. 990-993. http://dx.doi.org/10.1056/NEJM196505132721903

[8] C. E. Hayes, D. Kawatu, S. Mangray and N. S. LeLeiko, "Rectal Suction Biopsy to Exclude the Diagnosis of Hirschsprung Disease,” Journal of Pediatric Gastroenterology and Nutrition, Vol. 55, No. 3, 2012, pp. 268-271. http://dx.doi.org/10.1097/MPG.0b013e31824c0acc

[9] E. A. Ali, J. A. Morecroft, J. C. Bowen, J. Bruce and A. Morabito, "Wall or Machine Suction Biopsy for Hirschsprung's Disease: A Simple Modified Technique Can Improve the Adequacy of Biopsy,” Pediatric Surgery International, Vol. 22, No. 8, 2006, pp. 681-682. http://dx.doi.org/10.1007/s00383-006-1714-8

[10] N. J. Hall, D. Kufeji and A. Keshtgar, "Out with the Old and in with the New: A Comparison of Rectal Suction Biopsies with Traditional and Modern Biopsy Forceps," Journal of Pediatric Surgery, Vol. 44, No. 2, 2009, pp. 395-398. http://dx.doi.org/10.1007/s00383-006-1714-8

[11] J. Kessman, "Hirschsprung's Disease: Diagnosis and Management,” American Family Physician, Vol. 74, No. 8, 2006, pp. 1319-1322.

[12] B. I. Rees, A. Azmy, M. Nigam and B. D. Lake, "Complications of Rectal Suction Biopsy,” Journal of Pediatric Surgery, Vol. 18, No. 3, 1983, pp. 273-275. http://dx.doi.org/10.1016/S0022-3468(83)80098-0

[13] A. Pini-Prato, C. Carlini, F. Pesce, V. Jasonni and P. Seymandi, "Massive Bleeding after Rectal Suction Biopsy: Uncommon and Unexpected Delayed Onset," World Journal of Pediatrics, Vol. 7, No. 1, 2011, pp. 83-85. http://dx.doi.org/10.1007/s12519-011-0251-2

[14] J. A. C. Sterne and G. Davey Smith, "Shifting the Evidence-What's Wrong with Significance Tests?” Physical Therapy, Vol. 81, No. 8, 2001, pp. 1464-1469.

[15] R. Core Team "R: A Language and Environment for Statistical Computing,” R Foundation for Statistical Computing, Vienna, 2013. http://www.R-project.org/ 